the thymi of Stat3 $3^{-/}$mice than in wild-type mice, and destruction of the thymic architecture was evident. When thymcytes were removed from the Stat 3 mutant mice and exposed in vitro to apoptotic stimuli, the cells were no more sensitive to apoptosis than thymocytes from wild-type mice. So, the increased sensitivity of these cells is not due to an intrinsic defect, but rather due to a failure of the Stat $^{-/-}$TECs to protect them from apoptotic stimuli.

In conclusion, these results show that Stat 3 is vital in the maintenance of thymic architecture and for the survival of thymocytes. As far as the thymus goes then, Stat 3 is a protein you need if you want to maintain control of the ageing process.

Jenny Buckland

(4) References and links ORIGINAL RESEARCH PAPER Sano, S et al. Stat 3 in thymic epithelial cells is essential for postnatal maintenance of thymic architecture and thymocyte survival. Immunity 15, 261-273 (2001) FURTHER READING

Anderson, G. \& Jenkinson, E. J. Lymphostromal interactions in thymic development and function. Nature Rev. Immunol. 1, 31-40

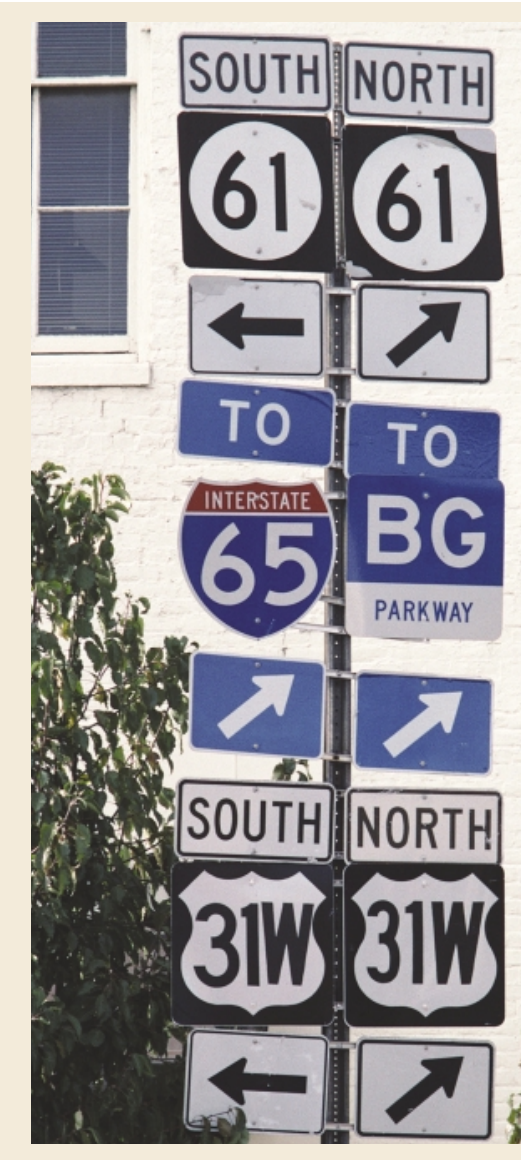

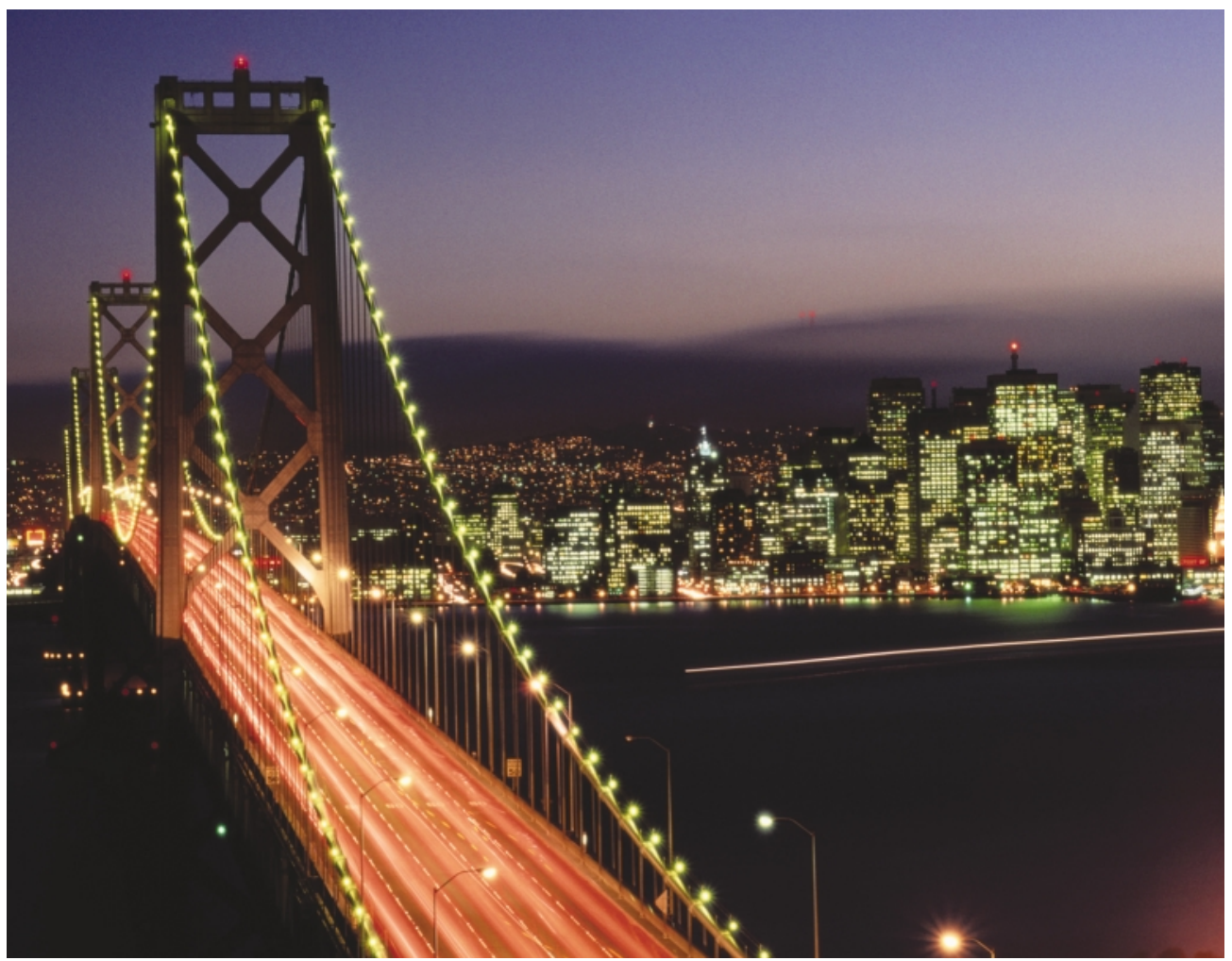

INNATE IMMUNITY

\section{New toll bridge}

The Toll-like receptors (TLRs) are emerging as the principal antigen sensors of innate immunity. In the earliest stages of infection, members of the TLR family discern the nature of the invader by detecting its conserved molecular patterns, and guide the ensuing adaptive response by inducing cytokines and costimulatory molecules. It is unclear how exactly this is achieved as all TLR-signalling components discovered to date are common to all TLRs and, indeed, interleukin-1 receptors (IL-1R). Two groups have now discovered a novel adapter molecule that specifically interacts with TLR4 and is a crucial bridge in the MyD88-independent signalling pathway.

MyD88 is a signalling adaptor containing a Toll/IL-1R (TIR) domain, a conserved motif that is essential for TLR signal transduction. Previous studies on MyD88-deficient mice, showed that this adapter is vital for signalling by TLRs, with the only known exception of TLR4 - a lipopolysaccharide (LPS) receptor - which can signal through both MyD88-dependent and MyD88-independent pathways. However, the adapter in this alternative, MyD88-independent pathway was not identified.

The new adaptor, described in the current studies, was identified in database searches for novel TIR domain-containing proteins. It is named TIR domain-containing adapter protein (TIRAP) by Horng et al., reporting in Nature Immunology; and MyD88-adapter-like (Mal), by Fitzgerald et al., reporting in Nature. Notably, unlike MyD88, TIRAP/Mal does not contain an amino-terminal death domain.

Both groups assessed TIRAP/Mal function in transfection studies, and showed that, similar to
MyD88, overexpression of TIRAP/Mal leads to activation of nuclear factor $\kappa \mathrm{B}(\mathrm{NF}-\kappa \mathrm{B})$. Is TIRAP/Mal, then, the elusive adapter of MyD88independent TLR signalling? To test this, both groups generated dominant-negative TIRAP/Mal mutants. The mutant TIRAP/Mal was shown to inhibit NF- $\kappa \mathrm{B}$ activation by TLR4, but did not interfere with NF- $\kappa$ B activation by TLR9 (the CpG receptor) or by IL-1R. Immunoprecipitation studies confirmed that TIRAP/Mal does indeed physically associate with TLR4.

To further investigate the functions of TIRAP/Mal, Horng and co-workers synthesized a peptide that corresponds with the predicted site of interaction with TLR4. This TIRAP/Mal peptide, attached to a delivery peptide that allows it to penetrate the cell membrane, inhibits LPS-induced, but not $\mathrm{CpG}$-induced, NF- $\kappa \mathrm{B}$ activation in a macrophage cell line. Furthermore, the TIRAP/Mal peptide was shown to inhibit LPS-induced, but not CpG-induced, upregulation of the co-stimulatory molecules CD80/CD86, cytokine production, and development of antigen-presenting capacity in dendritic cells.

Uncovering this new adapter is an important step in deciphering the complexity of TLR signalling, and determining which LPS-response genes are targets of MyD88- or TIRAP/Mal-dependent pathways must be a priority of future work.

(4) References and links

Jen Bell

ORIGINAL RESEARCH PAPERS Horng, T., Barton, G.M. \&

Medzhitov, R. TIRAP: an adapter molecule in the Toll signaling pathway. Nature Immunol. 2, 835-841 (2001) | Fitzgerald, K.A. et al. Mal (MyD88-adapter-like) is required for Toll-like receptor-4 signal transduction. Nature 413, 78-83 (2001)

FURTHER READING Akira, S., Takeda, K. \& Kaisho, T. Toll-like receptors: critical proteins linking innate and acquired immunity. Nature Immunol. 2, 675-680 (2001)

WEB SITES

Luke O'Neill's lab:

http://www.tcd.ie/Biochemistry/LONeill.html

Ruslan Medzhitov's lab:

http://info.med.yale.edu/immuno/fac_medzhitov.html 